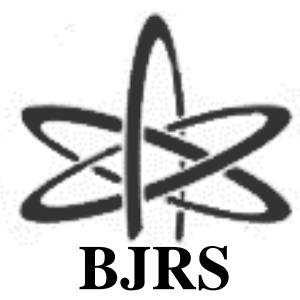
BRAZILIAN JOURNAL
$\mathrm{OF}$
RADIATION SCIENCES
09-02 (2021) 01-12

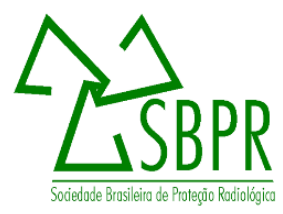

\title{
Methodology for correcting the nonlinear response of radionuclide calibrators
}

\author{
Quaresma $^{1}$ D.S., Oliveira ${ }^{2}$ A.E., Iwahara ${ }^{2}$ A. and Cunha ${ }^{3}$ P.G. \\ ${ }^{1}$ Observatório Nacional, 20921-400, Rio de Janeiro, RJ, Brazil \\ ${ }^{2}$ Instituto de Radioproteção e Dosimetria, 22783-127, Rio de Janeiro, RJ, Brazil \\ ${ }^{3}$ Universidade do Estado do Rio de Janeiro, 20550-900, Rio de Janeiro, RJ, Brazil \\ dansq@on.br
}

\begin{abstract}
Due to constructive and operational issues, the response of a radionuclide calibrator used in Nuclear Medicine can behave in a non-linear manner, especially in the transition of scales. Although the deviations from linearity are small, they may be important for standard secondary or reference radionuclide calibrators used in calibration laboratories. In the document TRS 454 - IAEA it is proposed that the deviation from linearity for these instruments should be in the range of $\pm 2 \%$. However, calibration laboratories may have some difficulty meeting the requirement. This article proposes an easy-to-implement methodology to correct the response of reference radionuclide calibrators from calibration laboratories, in order to meet the requirement of $\pm 2 \%$ for the linearity parameter recommended by the IAEA.
\end{abstract}

Keywords: Radionuclide calibrators, Nonlinear response, Linearity. 


\section{INTRODUCTION}

In nuclear medicine, it is important to measure the activity of radiopharmaceuticals before they are injected into patients to certify that the activity is the same as the one prescribed [1]. The instruments used to perform these measurements are radionuclide calibrator. The range of activities employed for diagnostic and therapeutic purposes is quite wide, ranging from a few MBq to the order of GBq. These activities generate currents in ionization chambers [2] ranging from fA to $\mu \mathrm{A}$. Thus, an electrometer associated with an ionization chamber must be capable of processing eight orders of magnitude of current. Thus, it is necessary for the electrometer to adjust its scales automatically. Discontinuities in the responses of the electrometers may occur in the transitions between scales [1].

Considering that the range of activities measured is wide and the radionuclide calibrator are calibrated with only one activity value $[3,4]$, it is important that the electrometer response be linear (the ratio between the measured activity and the conventional true activity must be constant over the entire range of currents provided by the activity-meter manufacturer). The International Atomic Energy Agency - IAEA [5] - recommends performance criteria for various metrological parameters. Different tolerance levels are proposed for field radionuclide calibrator, reference radionuclide calibrator and secondary-standard radionuclide calibrator. Regarding linearity, it is proposed for reference radionuclide calibrator and secondary-standard radionuclide calibrator that non-linearity be within the range of $\pm 2 \%$. This variable may represent an important contribution to measurement uncertainty. A methodology is proposed to correct the nonlinearity of the radionuclide calibrator' responses to meet the requirements proposed by the IAEA and to reduce the estimate of measurement uncertainty.

\section{MATERIALS AND METHODS}

The present study investigated the responses of CRC-15R and CRC-25R radionuclide calibrator manufactured by Capintec as a function of the activity of a ${ }^{99 \mathrm{~m}} \mathrm{Tc}$ source. All measurements were done at radionuclide calibrator ${ }^{99 \mathrm{~m}} \mathrm{Tc}$ setting. The activities were measured and recorded every two minutes. For the CRC-25R radionuclide calibrator, a source with an initial activity of $8.92 \mathrm{GBq}$ was 
used and measured for 79 hours. For the $\mathrm{CRC}-15 \mathrm{R}$ radionuclide calibrator, the source initial activity was $3 \mathrm{GBq}$, measured for 60 hours.

The result of each measurement $\mathrm{Mt}$ at time $\mathrm{t}$ was corrected for radioactive decay corresponding to a reference time $t_{0}$. For the $\mathrm{CRC}-25 \mathrm{R}$ radionuclide calibrator, $\mathrm{t}_{\mathrm{ref}}$ corresponds to the time at which $800 \mathrm{MBq}$ activity was recorded. This procedure has the objective of avoiding a possible effect due to ion recombination for higher activity values [2]. For the $\mathrm{CRC}-15 \mathrm{R}$ radionuclide calibrator, $\mathrm{t}_{\mathrm{ref}}$ corresponds to the beginning of the measurements.

Thus:

$$
M_{t_{c}}=M_{t} \times e^{\left(\ln 2 / T_{1 / 2}\right)\left(t-t_{r e f}\right)}
$$

The analysis of the values of $\mathrm{M}_{\mathrm{tc}}(\mathrm{t})$ allows the identification of the discontinuity points that represent the transition between the various electrometer scales of the radionuclide calibrator and the nonlinearity regions. Thus, it is possible to section the interval of measurements of the activity into $\mathrm{p}$ virtual scales. Each scale will be treated individually.

For each of the identified scales, the factor $f_{i}(A)$ is calculated, which will correct the discrepancy that occurred in the automatic scale changes of the electrometer, as well as the observed nonlinearity. Thus, for each scale $\mathrm{i}(\mathrm{i}=1,2, \ldots, \mathrm{p})$

$$
f_{i}(A)=\frac{M_{t_{m}}}{M_{t} \text { cal }}
$$

$\mathrm{M}_{\mathrm{t} \mathrm{m}}-$ measured activity

$\mathrm{M}_{\mathrm{t} \text { cal }}$ - calculated activity by decay constant value of Laboratoire National Henri Bequerel LNHB

Where $\mathrm{A}$ is the activity symbol and $\mathrm{M}$ is the value of the result of a measurement in MBq.

The function that best describes the data obtained by eq. 2 is:

$$
f_{i}(A)=a_{n} x^{n}+a_{n-1} x^{n-1}+\ldots a_{2} x^{2}+a_{1} x^{1}+a_{0}
$$

Where $\mathrm{n}$ is the degree of the polynomial. For the same data set, the degree $\mathrm{n}$ can assume several values. Due to the statistical characteristics of the data, the value of the correlation coefficient $\mathrm{R}^{2}$ of the fit may not be the most appropriate estimator for the selection of the optimal value of $n$. The 
residual sum of squares (SQE) has the largest amplitude of variation and is the most sensitive estimator to detect the appropriate degree of polynomial for fitting [6].

Thus, the criterion used was to determine $\mathrm{n}$ from which the SQE value shows no significant variations.

$$
\mathrm{SQE}=\sum_{\mathrm{j}=1}^{\mathrm{m}}\left(\mathrm{Y}_{\mathrm{j}}-\widehat{\mathrm{Y}}_{\mathrm{j}}\right)^{2}
$$

Where $Y_{j}$ is the value of the correction factor of the original data in measurement $j$; $\widehat{Y}_{j}$ is the value of the factor predicted by the fitted polynomial in the $\mathrm{j}$-th measurement.

\section{RESULTS AND DISCUSSION}

The values of $\mathrm{M}_{\mathrm{tc}}(\mathrm{t})$ obtained by eq. 1 are shown in figures 1 and 2 for the CRC-25R and CRC$15 \mathrm{R}$ radionuclide calibrator, respectively. The number of measuring points in figures 1 and 2 has been reduced for better visualization. For the CRC-25R radionuclide calibrator, 1 measuring point was used for every 10 samples and the CRC-15R radionuclide calibrator was used 1 measuring point per 30 samples. It can be observed that, for the CRC-25R radionuclide calibrator, at least one change of scale occurs at approximately $180 \mathrm{MBq}$, which corresponds to at least 34 hours. The two scales show non-linear behavior as a function of the activity value. The range from $8.92 \mathrm{GBq}$ to $180 \mathrm{MBq}$ was designated scale A. The second interval was designated scale B.

For the CRC-15R radionuclide calibrator, there are at least four changes in scale that occurred at approximately $1.72 \mathrm{MBq}, 56 \mathrm{MBq}$, and $12 \mathrm{MBq}$. The four scales show non-linear behavior as a function of the activity value. 


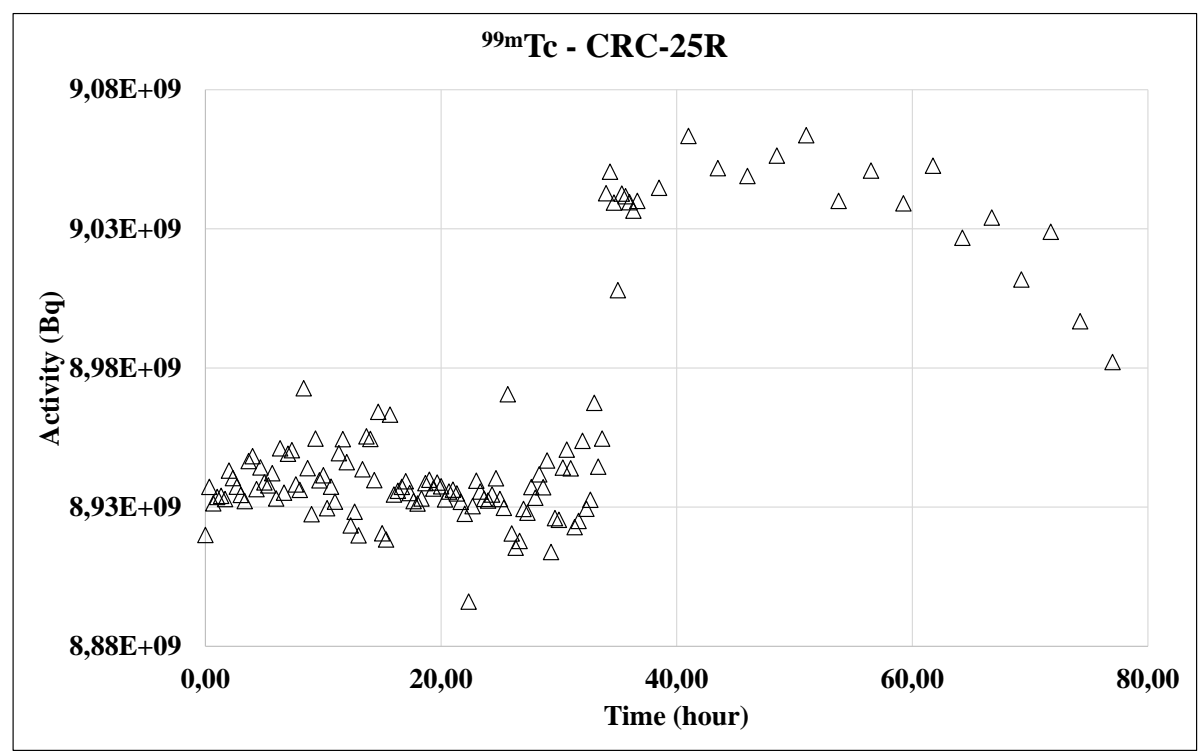

Figure 1: Corrected activity, $M_{t C}(t)$, measured in a ${ }^{99 m} T c$ source as a function of the time elapsed since the first measurement, for the CRC$25 R$ radionuclide calibrator.

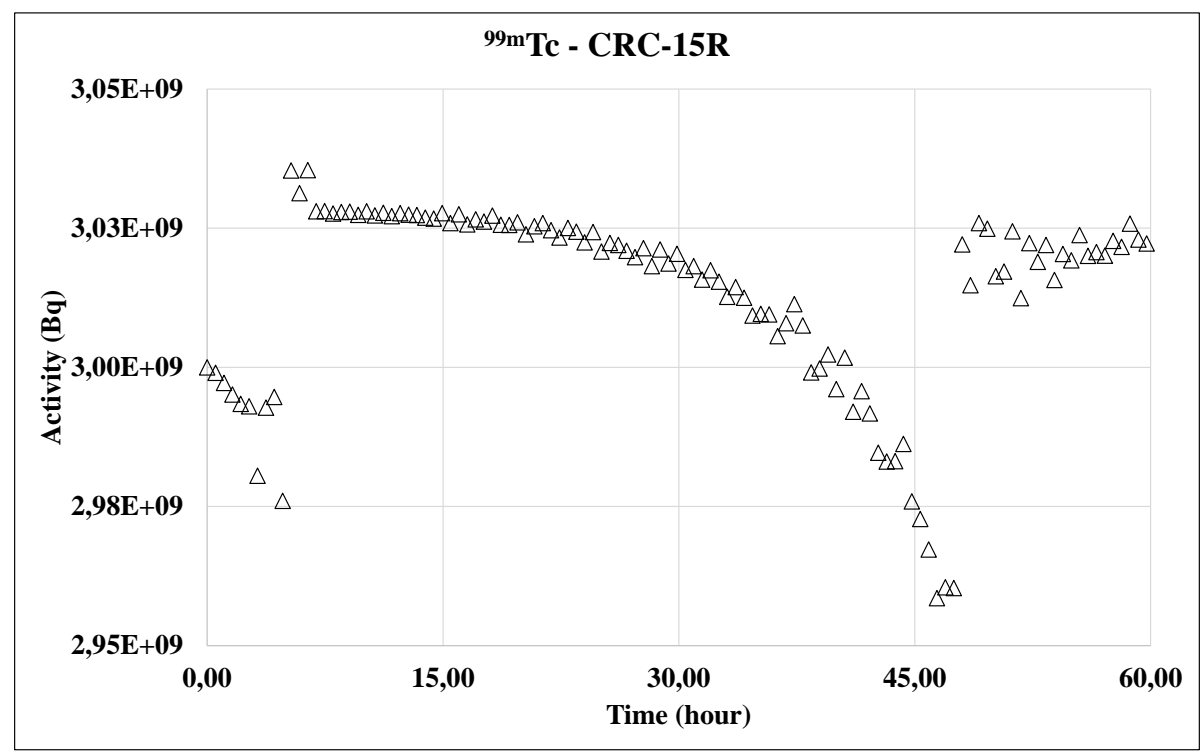

Figure 2: Corrected activity, $M_{t C}(t)$, measured in a ${ }^{99 m} T c$ source as a function of the time elapsed since the first measurement, for the CRC$15 R$ radionuclide calibrator.

For the CRC-25R and CRC-15R radionuclide calibrator, Figures 3 and 4 show, respectively, the experimental factors that correct the nonlinearity of their response. The functions $f_{i}$ were obtained by 
fitting using the least squares method. The degrees $n$ of the polynomials was selected using equation 4 by varying the $n$ values. Tables 1 and 2 show the results that lead to the determination of the optimal $\mathrm{n}$ values.

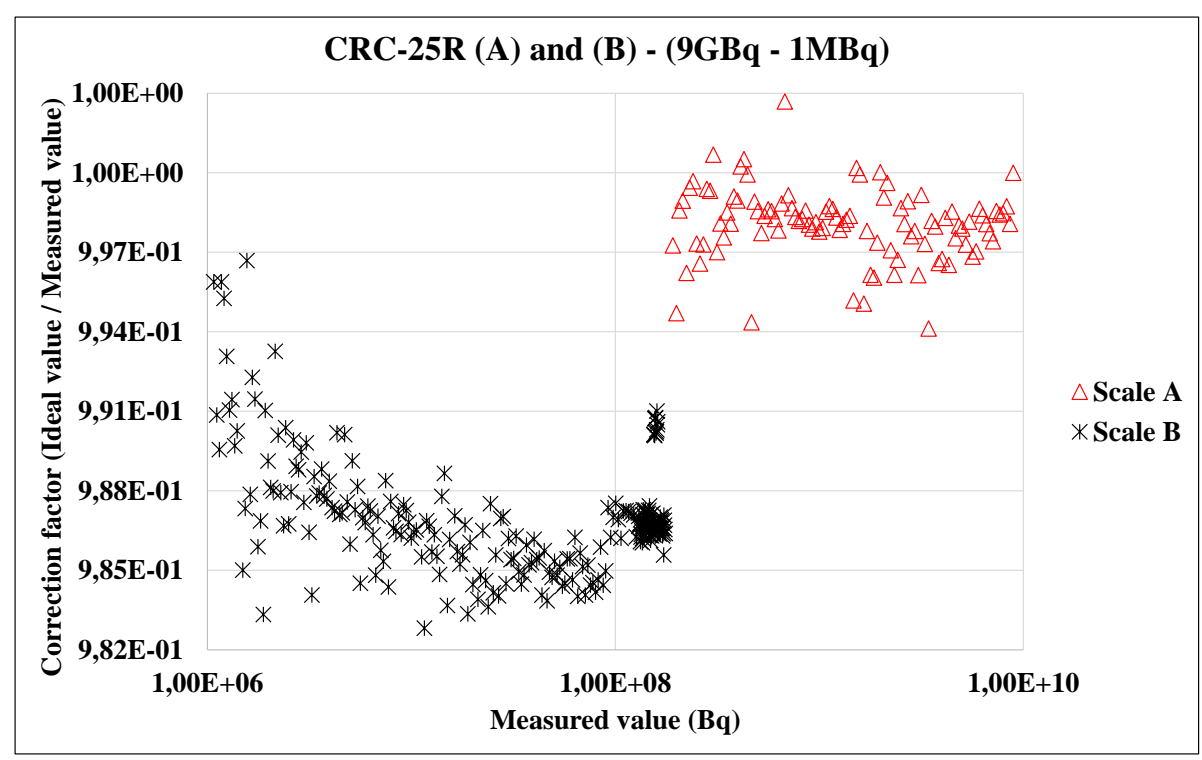

Figure 3: Nonlinearity correction factor for $A$ and $B$ scale of a $C R C$ $25 R$ radionuclide calibrator.

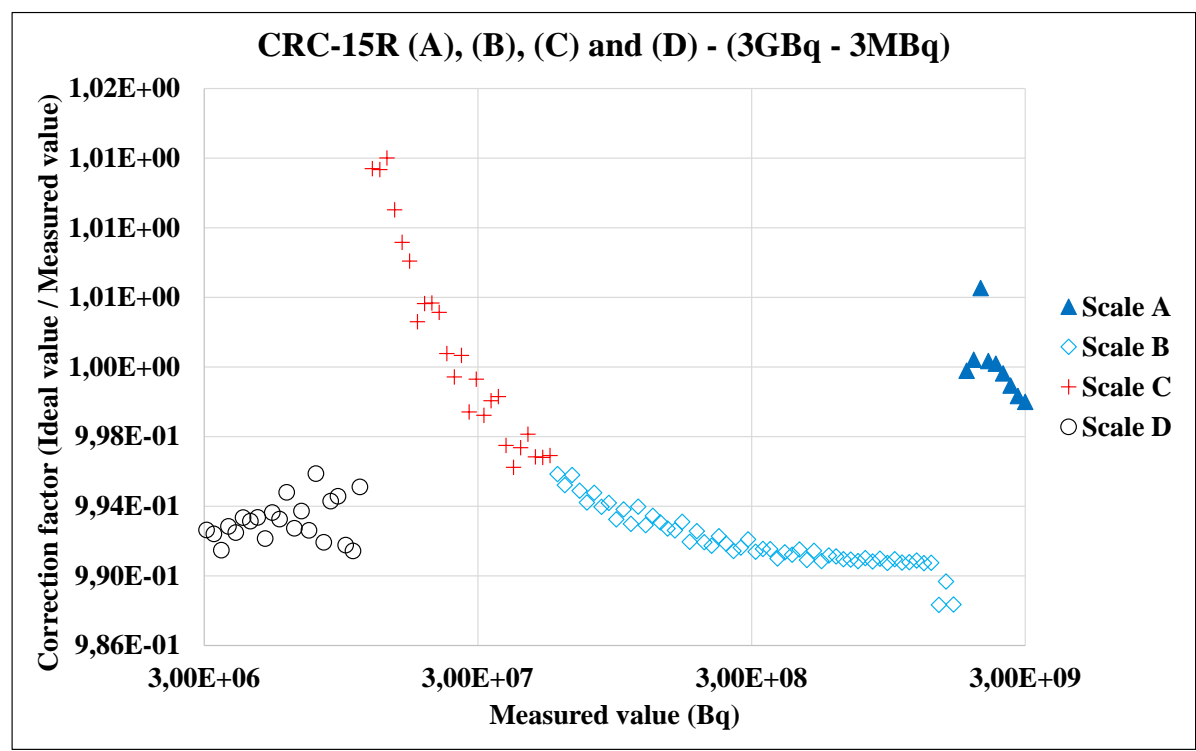

Figure 4: Nonlinearity correction factor for the $A, B, C$ and $D$ scales of a CRC-15R radionuclide calibrator. 
Table 1: Sum of squares of residuals (SQE) referring to various values of the degree of adjustment of the polynomial of the experimental data, for the scales $\mathrm{A}$ and $\mathrm{B}$ of the $\mathrm{CRC}-25 \mathrm{R}$ radionuclide calibrator.

\begin{tabular}{ccc}
\hline Degree of the & \multicolumn{2}{c}{ SQE } \\
\cline { 2 - 3 } polynomial & P(A) & P(B) \\
\hline 1 & $6.75 \mathrm{E}-02$ & $1.17 \mathrm{E}-03$ \\
2 & $\mathbf{4 . 2 7 E - 0 3}$ & $9.20 \mathrm{E}-04$ \\
3 & $4.26 \mathrm{E}-03$ & $7.56 \mathrm{E}-04$ \\
4 & & $7.33 \mathrm{E}-04$ \\
5 & & $\mathbf{6 . 7 2 E - 0 4}$ \\
6 & & $6.69 \mathrm{E}-04$ \\
\hline
\end{tabular}

Table 2: Sum of squares of the residuals (SQE) referring to various values of the degree of adjustment of the polynomial of the experimental data, for the A, B, C and D scales of the CRC-15R radionuclide calibrator.

\begin{tabular}{ccccc}
\hline $\begin{array}{c}\text { Degree of the } \\
\text { polynomial }\end{array}$ & \multicolumn{4}{c}{ SQE } \\
\cline { 2 - 5 } & $\mathbf{P}(\mathbf{A})$ & $\mathbf{P}(\mathbf{B})$ & $\mathbf{P}(\mathbf{C})$ & $\mathbf{P}(\mathbf{D})$ \\
\hline 1 & $7.28 \mathrm{E}-04$ & $2.15 \mathrm{E}-03$ & $3.42 \mathrm{E}-03$ & $\mathbf{2 . 0 1 E - 0 3}$ \\
2 & $7.15 \mathrm{E}-04$ & $1.18 \mathrm{E}-03$ & $1.15 \mathrm{E}-03$ & $2.01 \mathrm{E}-03$ \\
3 & $\mathbf{7 . 1 3 E - 0 4}$ & $7.60 \mathrm{E}-04$ & $8.72 \mathrm{E}-04$ & $2.01 \mathrm{E}-03$ \\
4 & $7.13 \mathrm{E}-04$ & $6.13 \mathrm{E}-04$ & $\mathbf{8 . 3 1 E - 0 4}$ & \\
5 & & $5.19 \mathrm{E}-04$ & $8.26 \mathrm{E}-04$ & \\
6 & & $\mathbf{4 . 8 5 E - 0 4}$ & & \\
\hline
\end{tabular}

The functions $\mathrm{f}_{\mathrm{ij}}$ for scales $\mathrm{A}$ and $\mathrm{B}$ of the $\mathrm{CRC}-25 \mathrm{R}$ radionuclide calibrator are:

Scale $\mathbf{A}=6.75 \mathrm{E}-23 \mathrm{x}^{2}-5.50 \mathrm{E}-13 \mathrm{x}+9.99 \mathrm{E}-01$

Scale B $=-2.67 E-42 x^{5}+1.21 E-33 x^{4}-2.01 E-25 x^{3}+1.50 E-17 x^{2}-4.82 E-10 x+9.90 E-01$

For the CRC-15R radionuclide calibrator, the functions are:

Scale $\mathbf{A}=1.84 \mathrm{E}-29 \mathrm{x}^{3}-1.37 \mathrm{E}-19 \mathrm{x}^{2}+3.31 \mathrm{E}-10 \mathrm{x}+7.42 \mathrm{E}-01$ 
Scale $\mathbf{B}=2.85 E-56 x^{6}-1.60 E-46 x^{5}+3.51 E-37 x^{4}-3.82 E-28 x^{3}+2.16 E-19 x^{2}-6.16 E-11 x+9.98 E-$ 01

Scale C $=1.37 \mathrm{E}-32 \mathrm{x}^{4}-2.21 \mathrm{E}-24 \mathrm{x}^{3}+1.36 \mathrm{E}-16 \mathrm{x}^{2}-3.99 \mathrm{E}-09 \mathrm{x}+1.05 \mathrm{E}+00$

Scale $\mathbf{D}=-1.17 \mathrm{E}-10 \mathrm{x}+9.94 \mathrm{E}-01$

For the CRC-25R and CRC-15R radionuclide calibrator, Figures 5 and 6 show, respectively, the original ${ }^{99 \mathrm{~m}} \mathrm{Tc}$ source measurement values corrected by radioactive decay and corrected by the function $f_{i j}$.

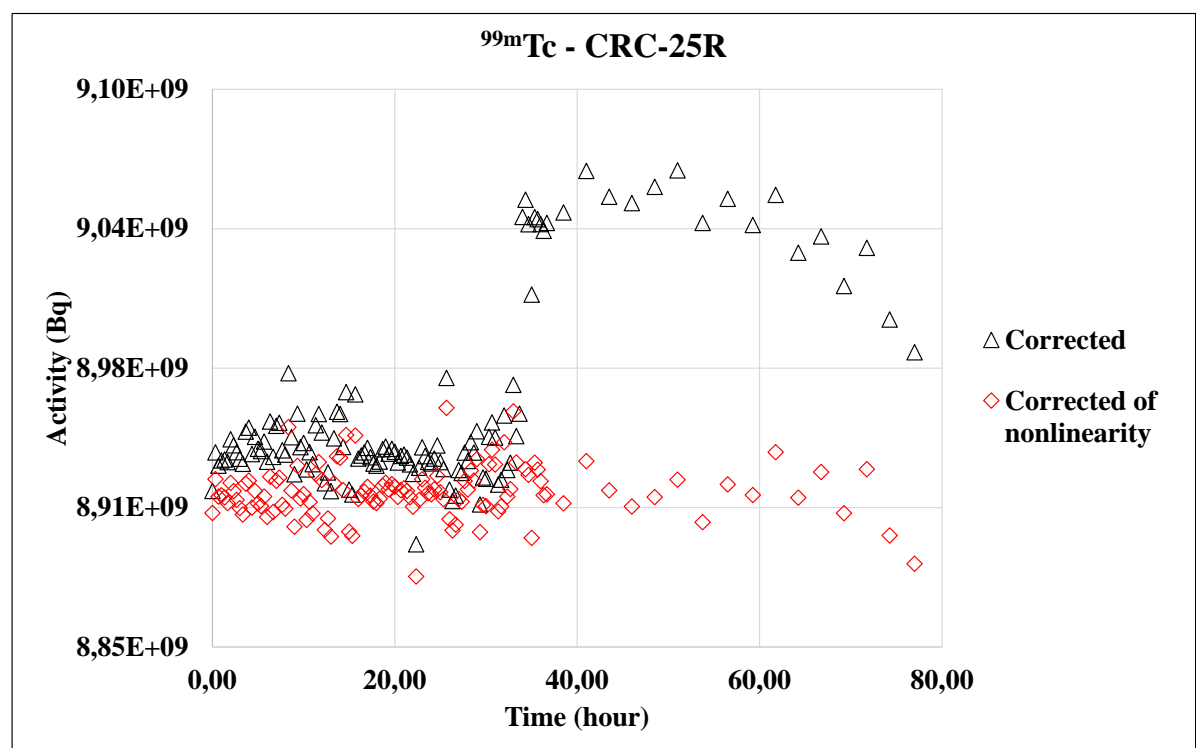

Figure 5: Experimental results of measuring $a^{99 m} T c$ source as a function of time and the same values after correcting nonlinearity, for the CRC-25R. 


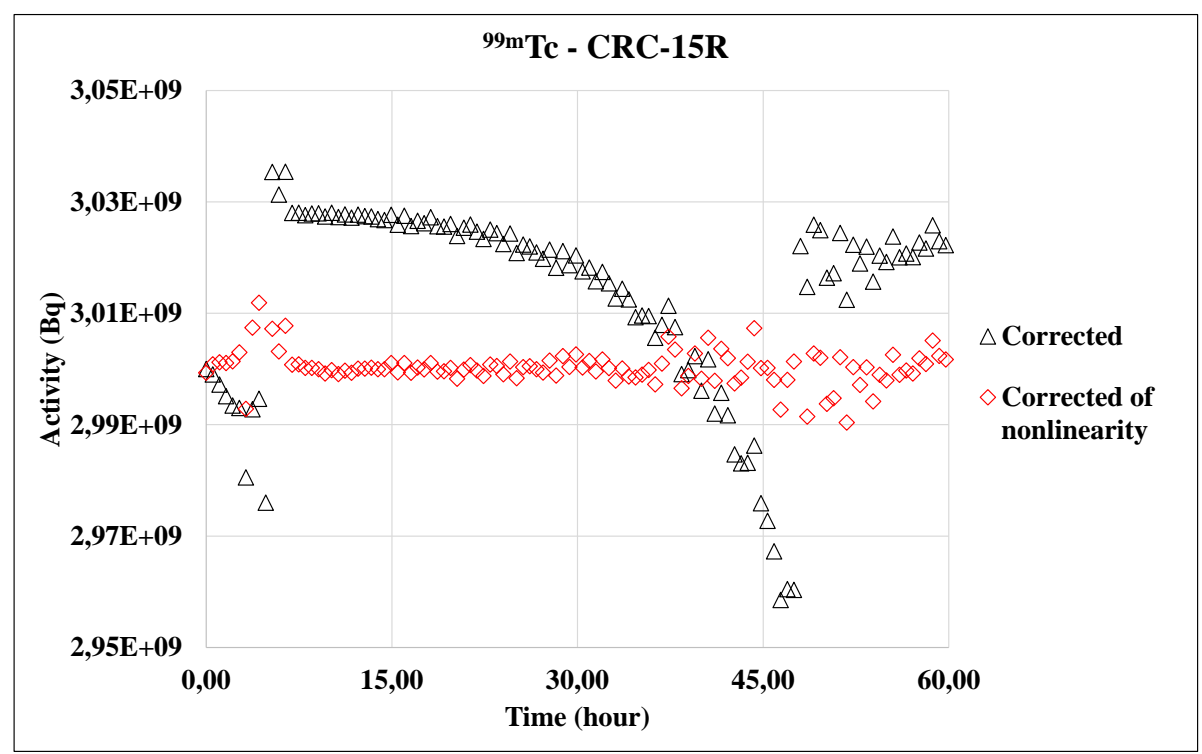

Figure 6: Experimental results of measuring $a{ }^{99 m} T c$ source as a function of time and the same values after correcting nonlinearity, for the $C R C-15 R$.

The range of variation of the residuals obtained after the nonlinearity correction is shown in Table 3.

Table 3: Range of variation of the residuals.

\begin{tabular}{ccc}
\hline $\begin{array}{c}\text { Linearity } \\
{ }^{\mathbf{9 9 m}} \mathbf{m}_{\text {Tc }}\end{array}$ & Lower and Upper limits of residuals - \% \\
\cline { 2 - 3 } CRC-15R & CRC-25R \\
\hline IAEA TRS 454 & $-2 / 2$ & $-2 / 2$ \\
Corrected & $-2.15 /+0.83$ & $-0.53 /+1.54$ \\
Corrected of nonlinearity & $-0.68 /+0.56$ & $-0.45 /+0.45$ \\
\hline
\end{tabular}

The methodology presented improves the linearity of the radionuclide calibrator responses as a function of the measured activity. At first, the better the proposition of the number $\mathrm{p}$, the better the correction. Figures 5 and 6 indicate that the methodology and the definition of the number $p$ of scales were able to significantly reduce the non-linearity of the responses of the radionuclide calibrator studied.

The range of variation of residuals relative to the difference between the experimental and theoretical data is significantly reduced after the correction of nonlinearity (Table 3 ). The values 
obtained are within the non-linearity tolerances recommended for reference radionuclide calibrator in the publication IAEA TRS 454 [5].

The methodology can additionally be evaluated by comparing the experimental values of the decay constant $\lambda$ before and after data correction with an internationally accepted theoretical value [7]. The corrected experimental data were fit by the least squares method for the function $\mathrm{LN}(\mathrm{A})=-\lambda \mathrm{t}+\mathrm{LN}\left(\mathrm{A}_{0}\right)$. The results of the fit are shown in Table 4.

Table 4: Statistics of linear regression of the response curves of the radionuclide calibrator before and after correction of the non-linearity.

\begin{tabular}{ccc}
\hline Statistics & CRC-15R & CRC-25R \\
\hline SQE $_{\text {before }}$ & 0.1722 & 0.0499 \\
SQE $_{\text {after }}$ & 0.0041 & 0.0050 \\
Standard uncertainty before & 0.0056 & 0.0038 \\
Standard uncertainty $_{\text {after }}$ & 0.0011 & 0.0020 \\
LN $\left(\mathrm{A}_{0}\right)_{\text {before }}$ & 21.8278 & 22.9114 \\
LN $\left(\mathrm{A}_{0}\right)_{\text {after }}$ & 21.8219 & 22.9116 \\
\hline
\end{tabular}

The value of the decay constant $\lambda$ of ${ }^{99 \mathrm{~m}} \mathrm{Tc}$ calculated from the corrected experimental data obtained by the both radionuclide calibrators studied coincides with the value adopted by LNHB [7] (Table 5) to the fifth significant digit. However, the difference between the values of $\lambda$ obtained before and after nonlinearity correction has little relevance for most practical situations. The difference between the activity values corrected by radioactive decay for $\mathrm{t}=10$ hours is only $0.2 \%$.

Table 5: Calculated values of the ${ }^{99 \mathrm{~m}} \mathrm{Tc}$ decay constant $\lambda$, before and after nonlinearity correction.

\begin{tabular}{ccc}
\hline Decay constant $\lambda\left(\mathbf{s}^{\mathbf{1}}\right)$ & CRC-15R & CRC-25R \\
\hline Without correction & 0.11545 & 0.11521 \\
With correction & 0.11540 & 0.11540 \\
LNHB & 0.11540 & 0.11540 \\
\hline
\end{tabular}




\section{CONCLUSIONS}

The results indicate that the nonlinearities of the studied radionuclide calibrator have less impact when compared to the levels of tolerance proposed by the IAEA [5] for Nuclear Medicine Services. However, it is important to make corrections when these instruments are used in Laboratories of Metrology as Reference Radionuclide calibrator for the purpose of calibrating another radionuclide calibrator used in a clinic. For a reference radionuclide calibrator, the corrections allow meeting the requirement of $\pm 2 \%$ recommended by the IAEA [5].

The method described in the present study presents a simple solution and allows a significant improvement of the measurement results over the entire range of the linearity curve studied, bringing the lambda decay constant and the half-life of the tested radionuclide to reference values [7].

\section{ACKNOWLEDGMENT}

One of the co-authors, Akira Iwahara, participated as a scholarship holder of Fundação de Amparo à Pesquisa do Estado do Rio de Janeiro - FAPERJ, whom we thank for his contribution in the development of this paper.

\section{REFERENCES}

[1] National Physical Laboratory, Protocol for establishing and maintaining the calibration of medical radionuclide calibrators and their quality control, A national measurement good practice guide NPL No. 93, 2006.

[2] Nuclear Medicine Physics. A Handbook for Teachers and Students. IAEA Pub 1617 (2015)

[3] Centro de Investigaciones Energéticas, Medioambientales y Tecnológicas - CIEMAT, Protocolo para la Calibración y el Uso de Activímetros.www.rdgroups.ciemat.es/web/lmri/serviciostecnicos, May, 3rd, 2021.

[4] Owner's Manual - CDC-25R, Capintec INC, Ver. H, 2015.

[5] International Atomic Energy Agency. Quality assurance for radioactivity measurement in nuclear medicine, TRS 454. Vienna 2006. 
[6] Applied Regression Analysis: A Research Tool, Second Edition, John O. Rawlings, Sastry G. Pantula, David A. Dickey (1998)

[7] LNE - LNHB/CEA Table des Radionucléides. C. Morillon, M. M. Bé, V. Chechev, A. Egorov (2012) 